\title{
A Cache Invalidation scheme through Data Classification in IVANET
}

\author{
Arun Kumar Dubey \\ School Of Information \\ Technology, RGPV \\ Bhopal, India
}

\author{
Dr. Sanjeev Sharma \\ School Of Information \\ Technology, RGPV \\ Bhopal, India
}

\begin{abstract}
Every one spends 10 to $40 \%$ time in vehicles a day that's why it is necessary to be updated with knowledge in vehicles. Vehicular Ad hoc Network (VANET) is a technology that establishes communication among vehicles through road side infrastructure. Internet Vehicular Ad hoc Network (IVANET) is a combination of wired internet and VANET technology. IVANET caches frequently access data items in a local storage of vehicles which have not storage problem. Due to high mobility of vehicles, a critical design issue is how to invalidate them when data items are updated and which data should be invalidated first. We have proposed an algorithm for data cache invalidation in IVANET as well as decide priority of data invalidation. In this scheme we classify invalidating data in to three categories queried data, emergency data and fixed data.
\end{abstract}

\section{Keywords}

VANET, Cache Invalidation Scheme, Invalidation Report

\section{INTRODUCTION}

A VANET is a technology that establishes communication among high speed mobile vehicles [1]. It facilitates vehicle to vehicle or vehicle to roadside infrastructure communication through a multi hop message relay without the assistance of any fixed infrastructure. Most of the concern of interest to MANET (Mobile Ad hoc Network) is interest in VANET but details differ. Rather than moving at random, vehicles move in organized way. The interactions with road side equipment can like wise characterize accurately and finally most vehicles are restricted in their range of motion for example by being constrained to follow paved highway.

In order to provide flexible connectivity, accessibility and a rich set of services, it is imperative to consider the integration of VANET with wireless infrastructure, such as wireless local area network and wireless wide area network. It is envisaged that IVANET will become a ubiquitous infrastructure in coming days.

A key optimization technique improving the communication performance of IVANET is to cache the frequently access data item in local storage. In an IVANET, it is less of problem to determine which data items to cache because memory in vehicle is not critically limited. When a data item in server is updated, it is necessary to invalidate cached copies of data item by broadcast invalidation report (IR). However, due to fast roaming vehicle, cache invalidation scheme develop for cellular network and mobile ad hoc network may not work well. Unlike these networks, energy conservation is not an issue in IVANET because vehicle is supported by vehicle power battery. Rather, our concerns are query delay in IVANET.

A number of cache invalidation schemes have been proposed previously, most of which is based on invalidation report (IR) $[7,8,9,10,11,12,13,14]$ and are targeting cellular network or mobile ad hoc network. A database server periodically broadcast an invalidation report, which includes list of update data items. Then mobile device invalidate cached data items. Previous invalidation schemes are based on energy conservation but vehicular ad hoc network has not this problem.

The following observations in IVANET characterize in IVANETs in the context of cache invalidation scheme: (1) High speed mobile vehicles can not reside in an area for long time. Therefore when data server broadcast IR, it is very difficult to recognize which coverage area should be target. Since multiple coverage areas are involved in a broadcast operation. (2) It is wasteful to broadcast same invalidation report to different vehicles, since most of content may not be relevant to them. (3) A web proxy caching may reduce network traffic [3] but it does not reduce network traffic in wireless links. In order to support a scalable caching operation with minimized invalidation report traffics in both data server and wireless network. It is necessary to co ordinate with network agent of location management.

To address these problems, we propose a scheme in which we try to effectively deal with cache invalidation of fast moving vehicles without incurring significant overhead. We contribute following work:

(a) We classify data in to three parts and schedule their priority in cache invalidation. This cache invalidation is performed through data server, home agent, access points and vehicles.

(b) These classified data are fixed data, queried data and emergency data. Fixed data are used at the time of new registration of vehicles. These data are coverage area information. Queried data are information asked by vehicles for specific purpose. Emergency data are information used for emergency services for example storms, Tsunamis etc.

(c) In the last, we give an IR based cache invalidation scheme which invalidates these three data.

\section{Cache Invalidation Sche mes}

In this section we analyze single cell and multi cell cache invalidation scheme. 
Single cell cache invalidation scheme is based on time stamp (TS) where base station broadcast invalidation report in regular interval time. Since IR is periodically broadcasted, there is unavoidable delay before answering question. This scheme can not be implemented in IVANETs because time stamp does not consider mobility of vehicles. For example when vehicles generate a query and wait for answer, they may miss IR if they moved to adjacent cell. Multi-cell cache invalidation scheme has reduced the limits of single cell cache invalidation scheme. Its design extended from asynchronous state full (AS) scheme [2]. In this scheme Mobile Switching Centre (MSC) located in higher network hierarchy than a Base Station (BS) execute cache invalidation operation. In VANET, vehicles exist in a coverage area for short period of time and travel along the large number of cells and thus, it is wasteful to examine cache invalidation with server on every hand off. Also, server's proactive IR transmission to multiple cells is not an efficient solution, if updated data item is not queried.

\subsection{Related Work}

In this section we explore Cooperative Cache Invalidation scheme (CCI), Enhanced Cooperative Cache Invalidation scheme (ECCI)[4] and on cache invalidation scheme by Sunho Lim[5]. In [4], data server maintains list of data items and access history by vehicles. This server does not blindly broadcast IR to vehicles, it sends IR to home agent and HA judiciously forward data through appropriate Gateway Foreign Agent (GFA). In this strategy GFAs do not proactively broadcast IR to individual vehicles, but reply the queries of vehicles on demand basis. CCI is a state-aware cooperative approach where data server and location management agents coordinate for data cache invalidation. In [5], authors demonstrated triangular routing based system on mobile IP. Here cache invalidation scheme is integrated with mobile IP location management system. Server asynchronously sends IR to HA rather than blindly broadcast to vehicles. Then HA judiciously refines data and distributes the IR to appropriate GFA based on triangular method based on mobile computing. Vehicles mobility is very high in VANET. When vehicle moves in to new coverage area within same regional network, it sends location update message to GFA. When vehicle moves in to different regional network, however, it sends location update message to HA through GFA and Access Point (AP). Both the scheme assumes all data are same for cache invalidation where as we classify data in to three parts and perform invalidation on these data differently. These data are fixed data, queried data and emergency data.

\section{Proposed Scheme}

In this section we discuss system model of proposed scheme, proposed approach and algorithm for proposed scheme.

\subsection{System Model}

In this system model all the location management is done by home agent. Previous models have used Gateway Foreign Agents (GFAs) but we removed these GFAs. In our hierarchical model, data server is on the top which is directly connected to home agent. The home agent is connected to access points. Each access point has its own coverage area. This system model has been demonstrated in figure 1 .

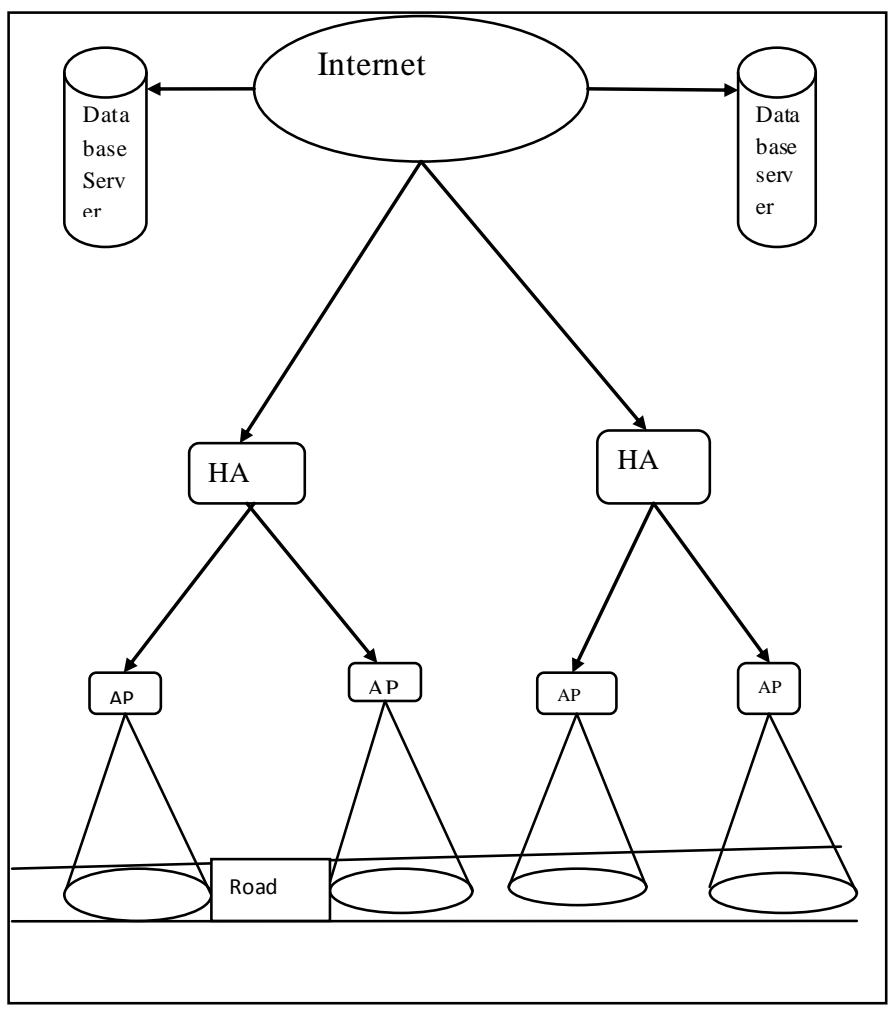

Fig 1: System model of proposed work

\subsection{Proposed Approach}

We classify data in to three parts for invalidation. Fixed data are invalidated through home agents. It contains complete information of network, coverage area and geographical information of regional network. These data do not disturb data server for invalidation. Queried data are invalidated through data server, HA and AP. Let us assume, in data invalidation, $40 \%$ data are fixed, $58 \%$ data are queried and $2 \%$ data are used for emergency. Emergency data invalidation is different from queried data. HA receive IR from server and broadcast in its network. These data have high priority in compare to other two data. As we have discussed $40 \%$ data are invalidated through HA hence vehicle does not need IR from server for $58 \%$ and $2 \%$. This reduces traffic at server and increase the bandwidth utilization for channel. Now IR from server is broadcasted to HA. HA at which vehicle is currently registered, passes IR to vehicle through access point. Rest of HA discard IR. Each vehicle is uniquely identified by mobile IP. Thus server does not have to keep record of vehicle.

\subsection{Cache Invalidation Algorithm}

Let the total number of data be $\mathrm{D}$ and total number of vehicle be N. then $40 \%$ data are fixed data $\left(F_{x}, x \in D\right), 58 \%$ data are queried data $\left(Q_{x}, x \in D\right)$ and $2 \%$ data are emergency data $\left(E_{x}, x\right.$ $€ D)$. Server will maintain a list of register $\left(R_{s}\right),\left[Q_{x}\right.$, vid $\left(V_{y}\right)$, $T_{x y}$, where $T_{x y}$ is time access of data $Q_{x}$ by vehicle id $V_{y}$. If queried data are changed the server will generate an $\mathrm{IR},\left\langle\mathrm{Q}_{\mathrm{x}}, \operatorname{vid}\left(\mathrm{V}_{\mathrm{y} 1}, \mathrm{~V}_{\mathrm{y} 2} \ldots\right), \mathrm{T}_{\text {curr }}\right\rangle$, and broadcast it to all HA. Here Tcurr is current time stamp and $\mathrm{V}_{\mathrm{y} 1}, \mathrm{~V}_{\mathrm{y} 2}$ are vehicle which access $Q_{x}$. Every vehicle must register with HA under which coverage area it exists. This is maintained in register $R_{h},\left[\operatorname{vid}\left(V_{y}\right)\right]$. On the receiving $I_{s}, H A$ compares $I_{s}$ and $R_{h}$. If 
match is not found IRs discarded, otherwise HA generates new IR $\left(\mathrm{IR}_{\mathrm{h}}\right)$ and judiciously send this new IR to vehicle through access points. In this way queried data are invalidated when data are updated at server.

Emergency data invalidating is different at HA. HA receives IR from data server and evaluates the type of data if it is found emergency data, broadcast $\mathrm{IR}_{\mathrm{h}}$ to its coverage area. Vehicles in this regional network are alarmed through emergency data invalidation. During this data invalidating HA interrupts other data invalidating and gives high priority to emergency data.

Fixed data invalidating perform through HA. HA does not disturb data server in this process. Now each fixed data is cached in vehicles register $\mathrm{R}_{\mathrm{v}}$, [id $\left.\left(\mathrm{HA}_{\mathrm{z}}\right), \mathrm{F}_{\mathrm{x}}\right]$ where $\mathrm{HA}_{\mathrm{z}}$ is home agent under which $\mathrm{Fx}$ was cached. HA broadcasts advertisement, [id $\left.\left(\mathrm{HA}_{\mathrm{z}}\right), \mathrm{T}_{\text {curr }}\right]$, at regular interval. As the vehicles enter new coverage area it will hear advertisement and send register request to new HA. Vehicle register matches id of $\mathrm{HA}_{\mathrm{z}}$ with register in the case of mismatch fixed data will be automatically invalidated.

Now vehicles request for new data, it sends a query packet, $<Q_{x}$, id $\left(\mathrm{HA}_{\mathrm{z}}\right)$, vid $\left(\mathrm{V}_{\mathrm{y}}\right), \mathrm{T}_{\text {curr }}>$, to server. Server updates its register with $\mathrm{Q}_{\mathrm{x}}$ and vid $\left(\mathrm{V}_{\mathrm{y}}\right)$. It replies to HAs with vehicle id and query packet. In this way our proposed work invalidating data in vehicles.

\section{Conceptual Analysis}

A conceptual analysis is done by us. We have assumed a road map in which 7 home agents (HAs), 30 access points (APs) and 250 vehicles exist. The network infrastructure would be connected by three protocols.

(a) Vehicle to Vehicle via ad hoc network.

(b) Vehicle to AP and AP to vehicle via wireless network.

(c) AP to server via wired TCP/IP connection network.

The following parameters have been used for analy sis:

\begin{tabular}{|l|l|}
\hline parameter & value \\
\hline Network size(km) & 24.5 \\
\hline Diameter of AP coverage(m) & 500 \\
\hline Diameter of car coverage(m) & 500 \\
\hline Velocity (km/h) & 60 \\
\hline Number of vehicles & 250 \\
\hline Database size (items) & 1000 \\
\hline Data item size (Kby te) & $1-500$ \\
\hline Hot data items & 100 \\
\hline
\end{tabular}

\begin{tabular}{|l|l|}
\hline Cold data items & Remainder of DB \\
\hline Hot data items access probability & 0.8 \\
\hline Number of HAs & 7 \\
\hline Number of APs & 30 \\
\hline
\end{tabular}

After using above parameters we have found significant result differences among Cooperative Cache Invalidation (CCI), Enhanced Cooperative Cache invalidation (ECCI) and proposed scheme. Proposed scheme has reduced the cost when query rate is high.

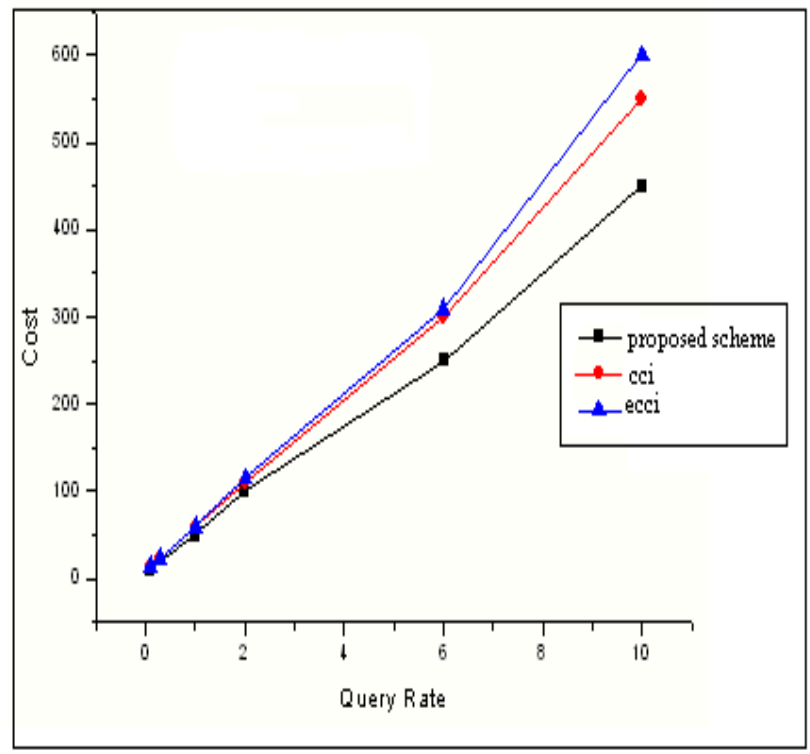

Fig 2: Query rate VS Cost

\section{Conclusion}

Our approach has considered to emergency data as a high priority data while cooperative cache invalidation scheme generated same IR for all data. We have tried to improve bandwidth utilization as well as reduced traffic over data server. We classified invalidating data in to $40 \%$ fixed data, 58\% queried data and $2 \%$ emergency data. This proposed scheme significantly improved the bandwidth utilization and query processing.

\section{REFERENCES}

[1] Dedicated Short range Communications (DSRC) Home. http://www.leearmstrong.com/D SRC/DSRCHomeset.htm.

[2] S. Khurana, A. Kohal, S. Gupta and P. Srimani”A strategy to manage cache consistency in distributed mobile wireless environment". In IEEE international conference on Distributed Computing Sy stem (ICDCS) 2000, pp 530-537. 
[3] H. Chen and Y. Xiao, "Cache Access and Replacement for Future Wireless Internet” IEEE communication magazine, pp 113-123, 2006

[4] Sunho Lim, Chansu Yu and Chita R. Das,"Cooperative Cache Invalidating Stratgies for Internet Vehicle Adhoc Network" IEEE 18th international conference, ICCCN 2009.

[5] Sunho Lim, Soo Roan Chae, Chansu Yu and Chita R. Das,"On Cache Invalidation For Internet Based Vehicular Ad hoc Network" 5th IEEE international conference on Mobile Ad hoc and Sensor Sy stems 2008, MASS 2008.

[6] C. E. Perkin, IP Mobility Support, Request For Comments (RFC) 2002-06.

[7] D.Barbara and T. Iimelinksi,"sleepers and Workaholics Caching Strategies for Mobile Environment",proc ACM sigmod,1994p.p.1-12

[8] G. Cao, A Scalable Low-Latency Cache Invalidation Strategy for Mobile Environments, IEEE transactions on knowledge and data en gineering, vol.15,no.5,sept/oct 2003.
[9] T.Hara"Effective Replica Allocation in Ad Hoc Networks for improving Data Accessibility"in proc,IEEE INFOCOM 2001,pp.1568-1576

[10] .....'Replica allocation in Ad hoc Networks with period data update"in proc $3^{\text {rd }}$ international conference on mobile data management.2002,pp 79-86.

[11] A.Kahol,S.Khurana, S.Gupta and P.Srimani,"An Efficient Cache management Scheme for Mobile Environment".in proc $20^{\text {th }}$ ICDS, April2000,pp530-537

[12] M. Saty anaray anan, J. Kistler, P. Kumar, M. Okasaki, E. Siegel, and D. Steere, Coda: A Highly Available File System For a Distributed Workstation Environment,IEEE Transactions on computers,vol.39,no.4,april 1990//23

[13] M. Kazar, Synchronization and Caching Issues in the Andrew File System, USENIX Conf., pp. 27-36,1988//17

[14] S. Sandberg, D. Goldberg, S. Kleiman, D.Walsh, and B. Lyon, Design and Implementation of the Sun Network File System, Proc. USENIX Summer Conf., pp. 119-130, June 1985 